\title{
A rare case of pseudo-quadricuspid aortic valve repair
}

Maureen Klepper, MD, Jama Jahanyar, MD, PhD, Gaby Aphram, MD, Laurent de Kerchove, MD, PhD, and Gebrine el Khoury, MD, Brussels, Belgium

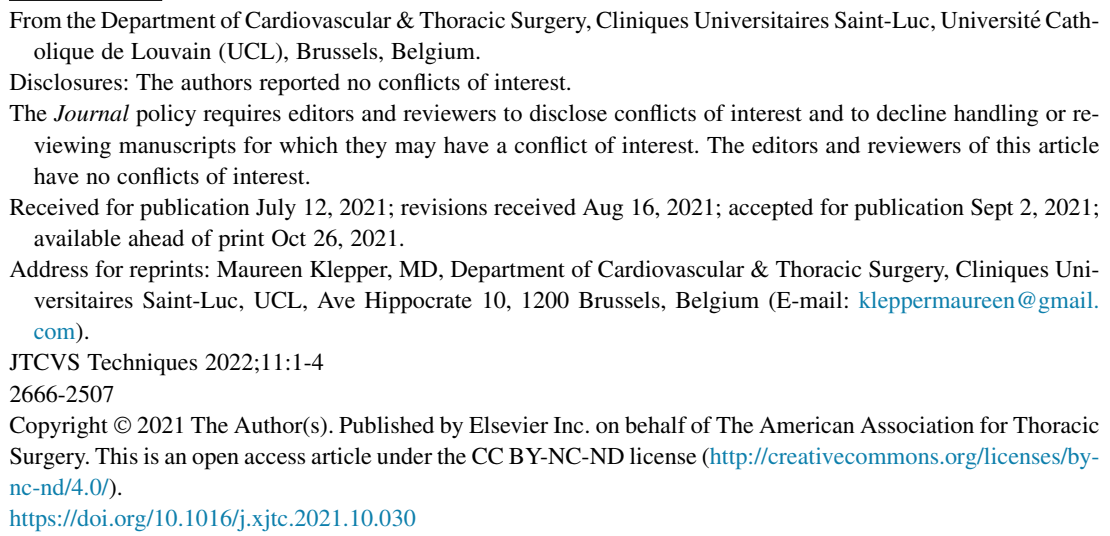
Video clip is available online.

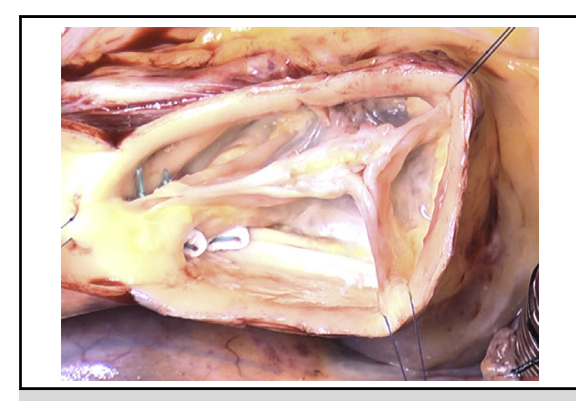

A repaired pseudo-quadricuspid aortic valve.

CENTRAL MESSAGE

Aortic valve phenotypes can vary substantially and require a tailored repair strategy, following general repair principles.

See Commentary on page 5 .
Quadricuspid aortic valves (QAVs) are rare congenital aortic valve phenotypes that are sometimes associated with other heart conditions. ${ }^{1}$ Coronary artery anomalies are the most commonly associated conditions and are present in up to $10 \%$ of patients, followed by pulmonary valve stenosis, subaortic stenosis, ventricular septal defect, nonobstructive cardiomyopathy, and aortic aneurysm. Several classifications have been proposed in the past. ${ }^{1,2}$ The first one was introduced by Hurwitz and Roberts in 1973, who described 7 subtypes (A-G) of QAV, based on the size of each cusp. ${ }^{1}$ Nakamura and colleagues ${ }^{3}$ developed a classification based on the location of the accessory cusp, and more recently, Vali and colleagues ${ }^{4}$ added a H-subtype to the original Hurwitz and Roberts classification, and, finally, Jagannath and colleagues ${ }^{5}$ simplified the Hurwitz and Roberts classification into 4 subtypes (I-IV).

Despite the many attempts to adequately classify this condition, it remains a rare aortic valve phenotype that is prone to aortic valve dysfunction and early deterioration with resultant aortic regurgitation. The patient population presenting with this valve phenotype tends to be younger and in the past would undergo aortic valve replacement as the standard of care. Nevertheless, valve repair can be a very good alternative to avoid prosthesis-related complications. We report a case of an aortic valve repair with concomitant ascending aorta replacement for a preoperatively diagnosed QAV with ascending aorta dilatation.

\section{CLINICAL VIGNETTE}

A 61-year-old man was referred to our institution for moderate aortic regurgitation, in the setting of an apparent QAV and severe ascending aortic dilatation. During his most recent follow-up, he was noted to have an increase in the size of his ascending aortic aneurysm from $48 \mathrm{~mm}$ in 2018 to $54 \mathrm{~mm}$ in 2021. The patient was asymptomatic, and his medical history was significant for diabetes mellitus type II, dyslipidemia, and he had a history of a transient cerebral ischemic attack in 2018. A transesophageal ultrasound scan confirmed moderate central aortic regurgitation related to the QAV with mild left ventricular dysfunction. The aortic root and annulus were not dilated. The coronary angiogram showed no significant coronary artery lesions. Hence, the patient was scheduled for an elective aortic valve repair with ascending aortic replacement.

\section{SURGICAL TECHNIQUE}

After a standard median sternotomy, the patient was placed on cardiopulmonary bypass via distal central aortic and right atrial venous cannulation. An aortic root vent was placed and the heart was arrested with warm blood cardioplegia. Subsequent cardioplegia after aortotomy was given every 15 minutes via direct cannulation of the coronary ostia. The ascending aorta was then opened $1 \mathrm{~cm}$ above the sinotubular junction (STJ) and retraction sutures were placed. The valve was then carefully assessed. Despite the 


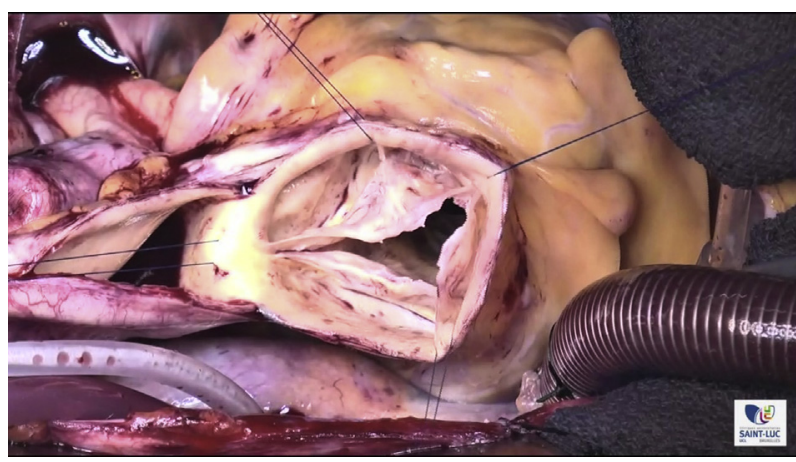

VIDEO 1. Repair of a rare pseudo-quadricuspid aortic valve. Video available at: https://www.jtcvs.org/article/S2666-2507(21)00721-5/fulltext.

preoperative echocardiographic assessment of the valve, which was consistent with a quadricuspid valve (defined as the presence of 4 cusps no matter their size but separated by 4 real commissures), during intraoperative assessment the valve appeared tricuspid with one non-coronary sinus with 2 cusps of equal size and 1 greater coronary sinus with 1 coronary cusp divided into 2 equal parts by a median raphe (hence, 3 real commissures and one nonfunctional commissure). Nevertheless, all cusp tissues were of good quality, with only localized fibrous thickening but no calcification. Hence, the valve was repairable (Video 1).

First, we removed the median raphe of the coronary cusp, by shaving of the cusp to increase its mobility. The fibrous thickening of the Noduli of Arantius and free margins were also shaven on the aortic side of the cusps, to improve their pliability and central coaptation of the valve. This was followed by a Cabrol annuloplasty at the non/left and inter-noncommissure using standard pledgeted $2.0 \mathrm{Ti}$ Cron sutures (Medtronic, Minneapolis, Minn) (Figures 1 and 2). The valve was then reassessed for symmetry, residual prolapse, and the length and height of coaptation. A water test demonstrated good central coaptation. We then proceeded with replacement of the ascending aorta with a $30-\mathrm{mm}$ prosthetic graft. The size of the prosthetic graft was determined by the height of the inter-noncommissure measured from the basal ring to the tip of the commissure. This measurement also corresponds to the diameter of the STJ. This is our standard technique to size a graft for aortic root replacement, and since the STJ is part of the functional aortic annulus (virtual basal ring to STJ), this also determines the graft required to stabilize the STJ. However, because in this case the aortic sinus was quite asymmetric, as we did not modify the entire functional aortic annulus, we verified our measurement with a ruler by measuring the diameter of the aortic root directly (Figure 2,E). This method, however, is not as reproducible and heavily depends on surgeon experience. Another method to size the STJ is by a Hegar dilator. However, we generally do not use a Hegar dilator due to fear of aortic valve injury. Only in cases of external aortic annuloplasty will we use a Hegar dilator, so we can precisely predetermine the size of the external annuloplasty. Interestingly, in our experience, the size of the ascending aortic graft generally falls between 26 and $30 \mathrm{~mm}$.
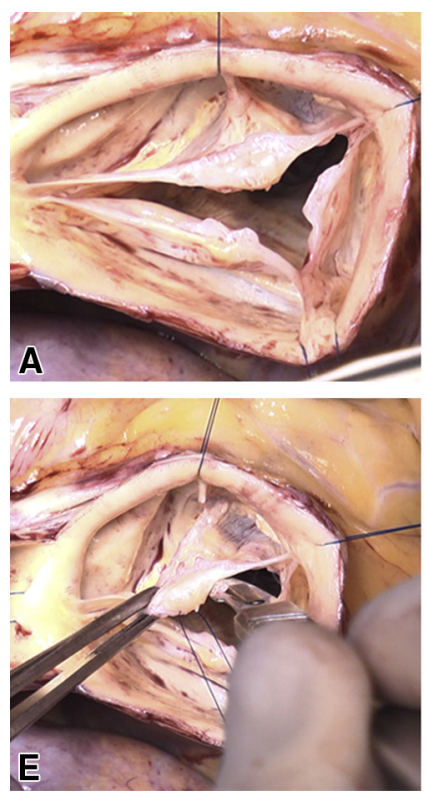
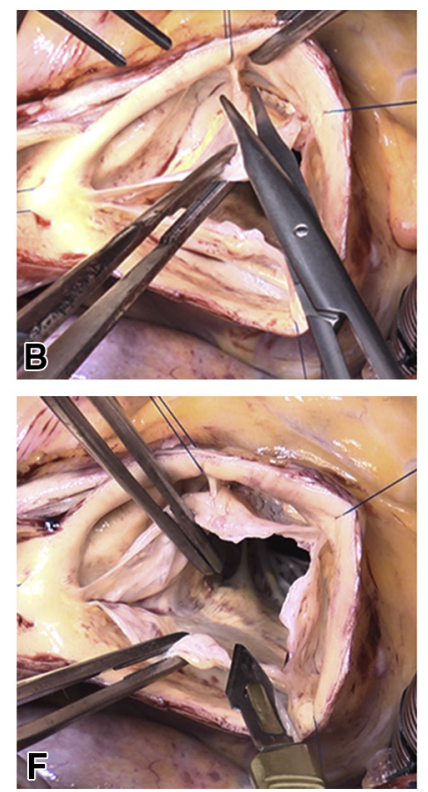
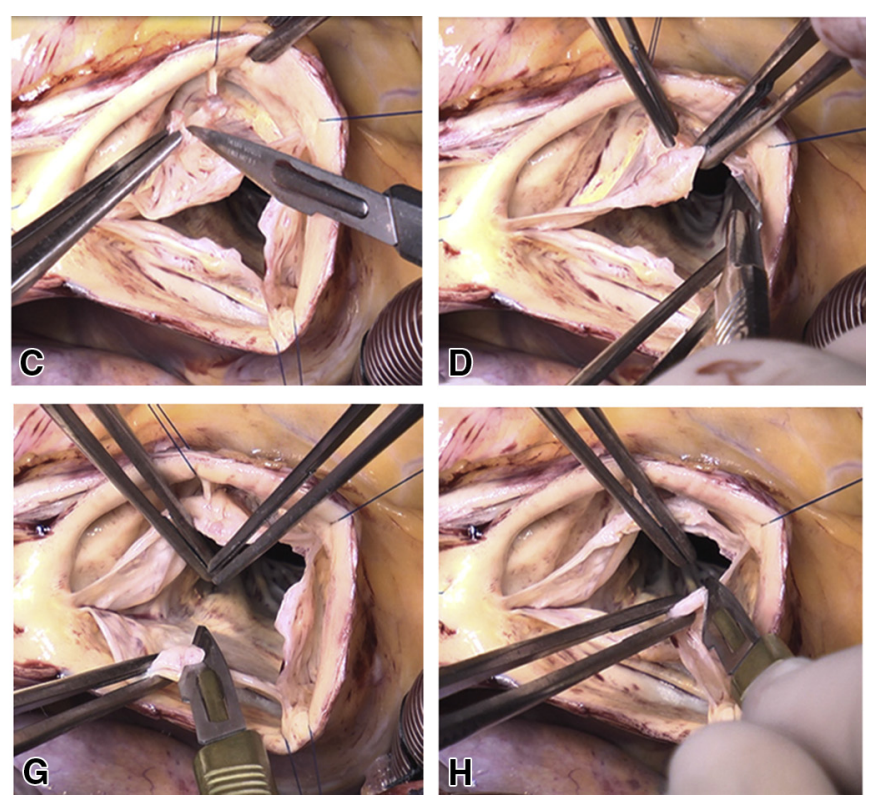

FIGURE 1. A, Intraoperative assessment reveals a tricuspid aortic valve with a bifid noncoronary cusp and a fused right/left coronary cusp with a central raphe. $\mathrm{B}$, Repair is begun by dividing the raphe toward the hinge-point of the conjoined cusp. C, The conjoined cusp is shaven to increase the pliability/ mobility of the cusp. D, A commissurotomy of mild fusion of the non/right commissure is performed with a number 11 blade. E, The nodules of Arantius and free margin of the conjoined cusp are shaved. F and G, The posterior noncoronary cusp is shaved. H, The anterior noncoronary cusp is shaved. 

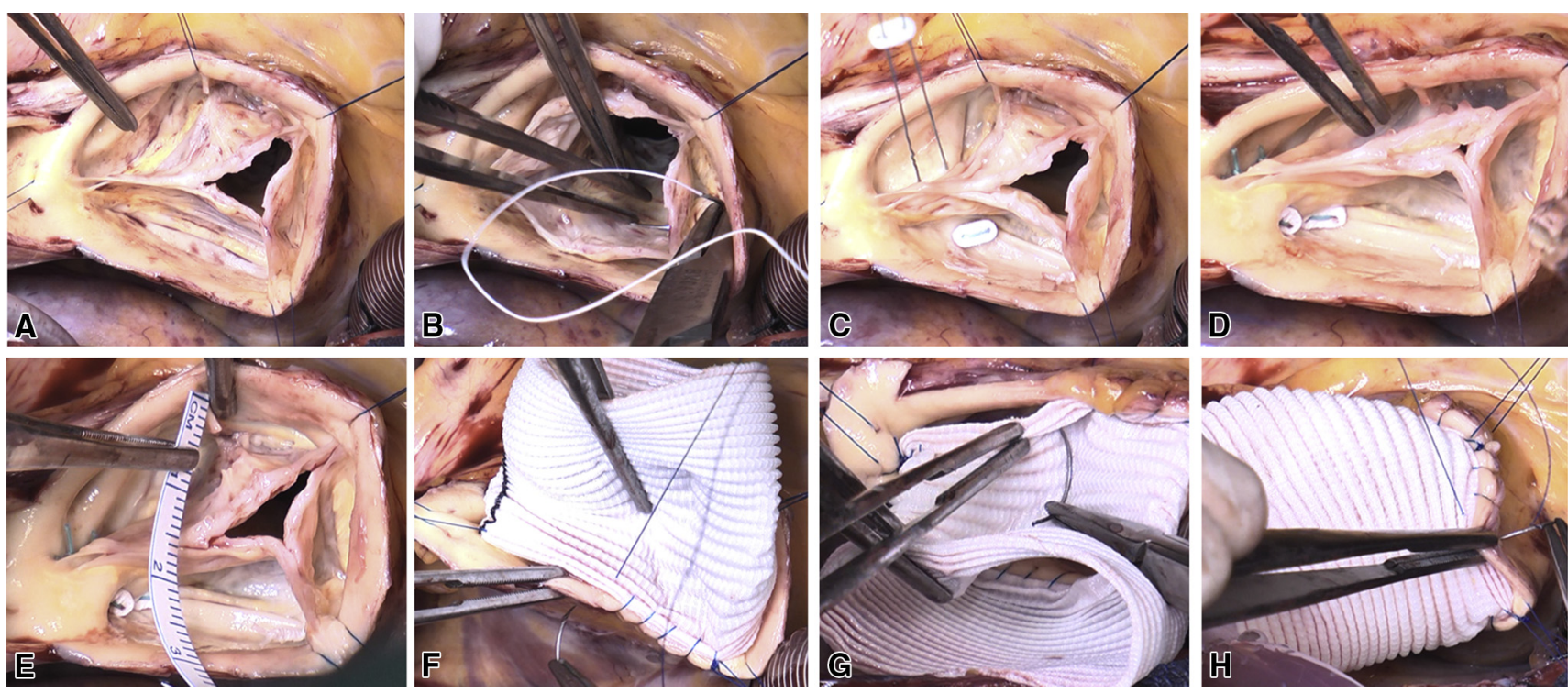

FIGURE 2. A, The pseudo-quadricuspid valve after leaflet modifications. B, Cabrol annuloplasty of the inter-noncoronary cusp commissure. C, Cabrolannuloplasty of the non/left commissure. D, A second Cabrol annuloplasty of the non/left commissure to improve annulus stabilization and leaflet coaptation. E, Sizing of the prosthetic graft by confirmatory direct measurement of aortic diameter with a ruler. F, Sewing of the Dacron graft to the sinotubular junction (STJ), from inter-noncommissure to non/left commissure, corresponding to the respective cusp. G, Sewing of the Dacron graft to the STJ between the non/right-commissure and non/left commissure. H, Sewing of the Dacron to the STJ, from the non/right- to inter-noncommissure, completing the proximal anastomosis and stabilizing the functional aortic annulus at the level of the STJ, respecting the valve geometry and its asymmetry.

Three commissural sutures were first passed through the graft respecting their particular orientation to avoid distortion of the repaired valve. The proximal anastomosis was performed with 4.0 polypropylene suture. After final check of the valve, the distal anastomosis was performed utilizing a short ring of the prosthetic graft to reinforce and wrap the distal ascending aorta. Written informed consent for publication was obtained from the patient. No institutional review board approval is required by our institution for case reports.

\section{COMMENTS}

QAVs are rare congenital anomalies that were first described in 1973 by Hurwitz and Roberts. ${ }^{1}$ Since then, several additional morphologic classifications have been proposed..$^{2-5}$

To the best of our knowledge, repair of this extremely rare variant of a tricuspid valve with quadricuspid- and bicuspid-like features has never been described before. We have named it pseudo-QAV, since it was difficult to categorize (it did not fit any of the known aortic valve phenotypes), but nevertheless appeared quadricuspid on preoperative echocardiogram. In our cumulative experience of more than 1000 aortic valve repairs, this was the first time we encountered this particular phenotype.

Because of unequal juxtaposition of the cusps in diastole and wall-stress, aortic dysfunction and early deterioration of the cusps can occur and lead to aortic regurgitation and need for surgical correction. Although mid- and long-term results of QAV-repair are relatively unknown, we believe that valve repair should be attempted if quality of tissues allows for it, especially in young patients considering the well-known complications of prosthetic valves. ${ }^{6,7}$ In this case, the repair outcome was excellent, and the postoperative course was unremarkable. The postoperative transthoracic echocardiography showed a competent tricuspid aortic valve with good central coaptation and a peak gradient of $4 \mathrm{~mm} \mathrm{Hg}$. The patient was hence discharged from the hospital on postoperative day 9. At our center, clinical and echocardiographic follow-up is routinely performed at postoperative month 1 and 6 , and then annually.

Rare aortic valve congenital anomalies, which fall out of the known spectrum of aortic valve phenotypes, constitute a surgical challenge for even experienced aortic valve repair surgeons. Of note, there are several learning points in this experience. It is tempting to bicuspidize this valve by sewing the bifid noncoronary cusps together, as it has been previously described for tricuspidization of a QAV. ${ }^{8}$ This would likely create a competent valve. However, this would also create several new problems. The first problem is that we now created a second abnormal cusp in addition to the already-existing abnormal fused cusp. Although the noncoronary cusp is bifid, we are still working with 2 rather normal cusps. In normal tricuspid aortic valves, the overall larger area of free margin length contributes to improved mobility and opening of the valve. By sewing the 2 edges 
of the noncoronary cusps together, the free margin length would decrease by close to $50 \%$, which in turn would also decrease valve mobility and create a new transvalvular gradient and possibly a clinically relevant stenosis. Moreover, there was still the possibility of altering the STJ and valve geometry during the proximal prosthetic graft anastomosis for the required ascending replacement, by not respecting the asymmetry of the native aortic valve. Nonetheless, the STJ is an important part of the functional aortic annulus, which also contributes to valve function, and thus requires meticulous remodeling with a correctly sized graft, while respecting the asymmetry and geometry of the native aortic valve (Figure 2, F-H). Hence, a solid understanding of these general principles will guide the surgeon to the correct repair strategy.

Although the feasibility and safety of QAV repair has previously been described, ${ }^{9}$ the gold standard for treatment of significant regurgitant QAVs still remains prosthetic aortic valve replacement. Nonetheless, our team believes that valve repair should be performed whenever possible, especially in young patients. QAVs are rare, and accordingly reported experiences are limited (early outcomes are excellent; mid- and long-term results are still pending). However, it is well established that aortic valve repair is far superior to prosthetic aortic valve replacement, with long-term survival equal to the general population. ${ }^{10}$ Results that have not yet been achieved with prosthetic aortic valves. Thus, we do not expect different results for QAVs. In our opinion and experience, especially in young patients, valve repair with close surveillance should be the preferred treatment modality for aortic regurgitation, irrespective of aortic valve phenotype.

\section{References}

1. Hurwitz LE, Roberts WC. Quadricuspid semilunar valve. Am J Cardiol. 1973;31: 623-6.

2. Cheng CL, Chang HH, Wang WC, Huang PJ, Lin SY. New morphological classification of congenital quadricuspid aortic valve and its histopathologic features. Cardiovasc Pathol. 2018:35:8-11.

3. Nakamura Y, Taniguchi I, Saiki M, Morimoto K, Yamaga T. Quadricuspid aortic valve associated with aortic stenosis and regurgitation. Jpn J Thorac Cardiovasc Surg. 2001;49:714-6.

4. Vali Y, Rajendra R, Nishtar S. A previously undescribed type of quadricuspid aortic valve: type H. J Heart Valve Dis. 2010;19:792-3.

5. Jagannath AD, Johri AM, Liberthson R, Larobina M, Passeri J, Tighe D, et al. Quadricuspid aortic valve: a report of 12 cases and a review of the literature. Echocardiography. 2011;28:1035-40.

6. Idrees JJ, Roselli EE, Arafat A, Johnston DR, Svensson LG, Sabik JF III, et al. Outcomes after repair or replacement of dysfunctional quadricuspid aortic valve. J Thorac Cardiovasc Surg. 2015;150:79-82.

7. Jamieson WR, Burr LH, Miyagishima RT, Germann E, Macnab JS, Stanford E, et al. Carpentier-Edwards supra-annular aortic porcine bioprosthesis: clinical performance over 20 years. J Thorac Cardiovasc Surg. 2005;130:994-1000.

8. Mastrobuoni S, Aphram G, Tamer S, Navarra E, De Kerchove L, El Khoury G. Quadricuspid aortic valve repair. Ann Cardiothorac Surg. 2019;8:433-5.

9. Daprati A, Generali T, Arlati F, Roberto M. Quadricuspid aortic valve plasty: is it worth it to repair as an alternative to substitution? Ann Thorac Surg. 2013;95:e7-8.

10. de Meester C, Vanovershelde JL, Jahanyar J, Tamer S, Mastrobuoni S, Van Dyck M, et al. Long-term durability of bicuspid aortic valve repair: a comparison of 2 annuloplasty techniques. Eur J Cardiothorac Surg. 2021;60:286-94. 